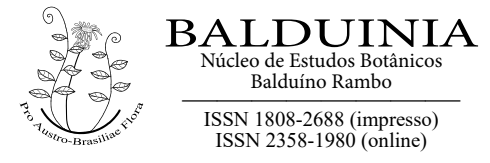

BALDUINIA, ano XVII, nº 67: 21-25. 2021.

Publicado em 4 junho de 2021.

DOI: $10.5902 / 2358198066127$

\title{
Updates on geographic range and synonymies in species of Podocoma (Asteraceae: Astereae)
}

\author{
Leonardo Paz Deble ${ }^{1,2}$
}

\begin{abstract}
Resumo. A distribuição geográfica de Podocoma Blanchetiana inclui campos arenosos (região dos Campos de Areia), entre os municípios de Alegrete, Manoel Viana e São Francisco de Assis, sudoeste do estado do Rio Grande do Sul, Brasil. Além disso, Stenachaenium megapotamicum var. pedunculata e St. megapotamicum f. minor são adicionados à sinonímia de $P$. hieracifolia. São designados lectotipos para St. megapotamicum f. minor e Podocoma Blanchetiana var. intermedia.

Palavras-chave: Brasil, Paraguai, Podocoma, Rio Grande do Sul, Stenachaenium.
\end{abstract}

\begin{abstract}
The geographic range of Podocoma Blanchetiana includes the sandy grasslands (Campos de Areia region), in Alegrete, Manoel Viana and São Francisco de Assis municipalities, southwest Rio Grande do Sul state, Brazil. Furthermore, Stenachaenium megapotamicum var. pedunculata and St. megapotamicum f. minor, are added to the synonymy of $P$. hieracifolia. Lectotypes are designated to St. megapotamicum f. minor and Podocoma Blanchetiana var. intermedia.

Key words: Brazil, Paraguay, Podocoma, Rio Grande do Sul, Stenachaenium.
\end{abstract}

Podocoma Cassini (1817: 137) comprises only five species distributed mainly in southeast South America, and occurs in Argentina, Brazil, Bolivia, Paraguay and Uruguay. The species grow on open environments, mainly on grasslands, savannas, mountainous places, and rocky (Sancho et al. 2010). Morphological attributes to recognize the genus Podocoma comprises the habit herbaceous, the subterranean rhizomes, the stem single or rarely branched only in the distal third, the heads solitary or few, compounding a corymbose capitulescence, the phyllaries arranged in 4-5 series, being the outermost phyllaries smaller than the innermost phyllaries, all acute or acuminate at apex, the ray flowers pistillate, with corolla shortly ligulate, white or white-cream in color (purplish at the end of the anthesis), and with ligula slightly exceeding the involucre, the disc-flowers perfect, with corolla yellow and limb five-lobed, and mainly by its cypselae strongly compressed, elliptic in shape, narrowed at apex into a conspicuous neck, with the margins ribbed, and epidermis villous, being covered by short twin-trichomes (Baker 1882, Nesom 1994, Sancho et al. 2010).

Podocoma was revised in different moments since its description by Cassini $(1817,1826)$, including the revisionary studies proposed by Candolle (1836), Bentham (1873), Baker (1882), and the description of new species (Hochreutiner 1899, Cabrera 1932, Malme 1933), and more recently, the generic treatment proposed by Nesom \& Zanowiak (1994), which update the number of species to eight, with the inclusion of two species before recognized in Conyza, and, finally, the last review of Podocoma proposed by Sancho et al. (2010), which present a generic reassessment and stablish in five the number of valid taxa. Even with all these contributions, based in recent collections performed in Rio Grande do Sul state, Brazil, and in the study of nomenclatural types, some novelties are evidenced including the update in the geographic distribution of Podocoma Blanchetiana and the placement of two taxa in the synonymy of $P$. hieracifolia. All valid species treated are described, and comments about geographic distribution, taxonomic relationships are also presented.

\section{Results and Discussion}

Podocoma Blanchetiana Baker, Fl. Bras. 6 (3): 15. 1882. Type: BRAZIL. Bahia, "Habitat in prov. Bahia ad Igregia Velho", 1845, J.S. Blanchet

\footnotetext{
Accepted on January 2, 2021.

${ }^{1}$ Universidade Federal do Pampa - Unipampa campus Dom Pedrito, Rua 21 de abril 80, CEP 96450-000. E-mail: deble.biol@gmail.com (author for correspondence).

${ }^{2}$ Núcleo de Pesquisas Botânicas Balduíno Rambo, Universidade Federal de Santa Maria, Av. Roraima, CEP 97105-900.
} 
3365 (lectotype, designated by Sancho et al. (2010: 498): BR0698188 photo!, isolectotypes: BM000901153 photo! HAL0139374 photo! K000052857 photo! NY00232548 photo! P00578754 photo! P00578755 photo! P00578759 photo! KW001001828 photo!). Figure 1B, C.

Perennial herbs, 15-35 cm high; rhizome developed, with long and straight roots; stem solitary, erect-ascendant or erect, not ramified or 2-3 branched in distal third, densely to laxly pilose, covered by flagelliform trichomes, with 1100$2400 \mu \mathrm{m}$ long. Leaves at base concentrate, then reduced, and the most distal bracteiform; blades chartaceous in texture and not or slightly discolor. Proximal leaves oblanceolate, oblong or obovate, $4-9.5 \times 1.3-4 \mathrm{~cm}$, base attenuate, apex slightly acute, one principal vein and 1-3 lateral veins, one medial and two lateral more or less basal, margins shortly denticulate, sparsely to densely pilose on both surfaces, covered by flagelliform trichomes, often yellowish or ochre, and up to $1800 \mu \mathrm{m}$ long; distal leaves reduced, oblong or elliptic, 1.5-4 $\times$ $0.5-1.5 \mathrm{~cm}$, the most distal bractiform, ellipticlanceolate or lanceolate, $0.5-1.5 \times 0.2-0.5 \mathrm{~cm}$. Heads disciform, solitary or up to 3 , peduncles $1.5-8 \mathrm{~cm}$ long, densely pilose; involucre 9-11 $\mathrm{mm}$ high, campanulate; phyllaries 3-4-seriate, gradate, margins pale, dorsally greenish and tips purplish when dry; outer phyllaries 5-7 × 0.7$0.9 \mathrm{~mm}$, narrowly elliptic-lanceolate or linearlanceolate, acute, slightly pilose; middle and inner phyllaries $8-12 \times 0.8-0.9 \mathrm{~mm}$, linear-elliptic, acute, dorsally scarcely pilose, margins deeply fimbriate at apex; receptacle subconvex, deeply alveolate. Ray flowers in 2-3-series, white-cream, then purplish; corolla 7-7.5 mm long, tube 2.2-3 $\mathrm{mm}$, oblong, glabrous; style branches dorsally glabrous. Disc-flowers numerous, perfect, corolla 8-8.5 mm long, hairy with an ampliated throat, at apex 5-dentate, teeth $0.5-0.8 \mathrm{~mm}$; anthers c. $2 \mathrm{~mm}$ long, thecae auriculate at base, apical appendage oblong, subobtuse; style branches 1.8-2.0 mm long, dorsum with sweeping trichomes. Cypselae elliptic, 5.5-7.5 $\mathrm{mm}$ long, margins ribbed, narrowed at apex into a neck, neck 2-3 mm long; pappus $c$. 7-9 $\mathrm{mm}$ long, bristles unequal.

Etymology- Baker (1882) dedicated this species in honor to Jacques Samuel Blanchet (1807-1875), Swiss botanist, who collected in northeastern Brazil (Bahia).
Material examined - BRAZIL. Rio Grande do Sul: Manoel Viana, trevo de acesso a São Francisco de Assis, em palmar de Butia lallemantii, Nov 2019, L.P. Deble \& B.P. Moreira 19004 (PACA!).

Discussion - Despite with few collections registered in Herbaria, Podocoma Blanchetiana shows a wide geographical distribution, occurring from Bahia to northeastern Argentina. In Brazil, the most southern collection points until this moment were in Santa Catarina state, on high grasslands among rocks. The material found in Rio Grande do Sul state occurs on sandy grasslands in the Campos de Areia region (Figure 1A). The population registered in Rio Grande do Sul is the same ecotype registered in Corrientes and Misiones provinces, northeast Argentina, due its large leaves, with blades covered by abundant and long flagelliform trichomes.

Podocoma hieracifolia (Poir.) Cassini, in Cuvier Dict. Sci. Nat. 42: 60. 1826. Basyonim: Erigeron hieracifolius Poir. [as hieracifolium], in Lamarck Encycl. 8: 491. 1808. Type: ARGENTINA. Buenos Aires: Commerson s.n. (holotype FI006219 photo!). =Podocoma primulifolia Cass., in Cuvier Dict.

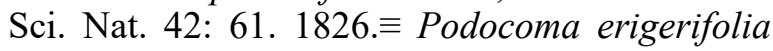
Steud., Nomenc. Bot. (ed. 2) 1: 584. 1840. Type: URUGUAY. Montevideo: 'Montevideo, 1767', May 1767, Commerson s.n. (holotype P00506878 photo!).

=Podopappus pubescens Hook. \& Arn., Companion Bot. Mag. 2 (14): 50. 1836. Type: ARGENTINA. Buenos Aires: "Buenos Ayres, s.d." Tweedie s.n. (holotype K000221722 photo!). =Stenachaenium megapotamicum var. pedunculata Chodat, Bull. Herb. Boissier, ser. 2, 2: 388. 1902. Type: PARAGUAY. San Pedro: "in regione vicine San Estanislao in campo" E. Hassler 4277 (holotype G not seen, isotypes P02698023 photo! P02698024 photo!) syn. nov.

$=$ Stenachaenium megapotamicum $\mathrm{f}$. minor Chodat, Bull. Herb. Boissier, ser. 2, 3: 717. 1903.Type: PARAGUAY. Río Apa, Nov 1902, E. Hassler 7831 (Lectotype (here designated) G00448740, isolectotypes: NY00232550 photo!, S20-1320 photo!) syn. nov.

= Podocoma blanchetiana var. intermedia Hassl., Repert. Spec. Nov Regni Veg. 16 (1-4): 27.1919. Type: PARAGUAY. Río Apa, Nov 1902, E. Hassler 7831 (Lectotype (here designated) G0448740, NY00232550 photo! S20-1320 photo!). 
Deble, L.P., Updates in Podocoma.

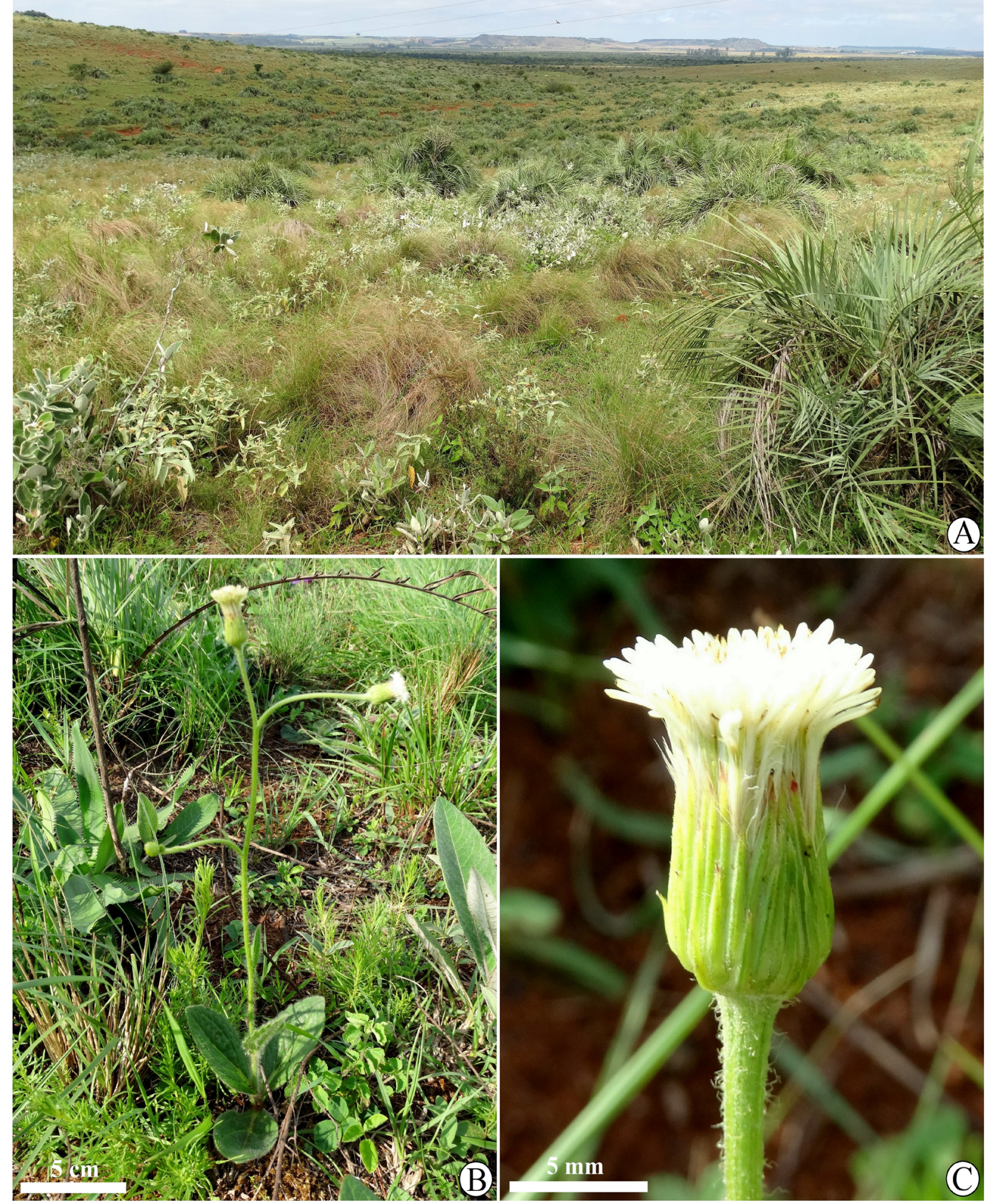

Figure 1. Podocoma Blanchetiana. A. Local of ocorrence, in the Campos de Areia region. B. General aspect. C. Capitulum (B-C from Deble \& Moreira 19004). 
Perennial herbs, 15-50 cm high; rhizome developed, with long and straight roots; stem solitary, erect-ascendant or erect, not ramified or 2-3 branched in distal third, densely to laxly pilose, covered by conical trichomes, with up to $800 \mu \mathrm{m}$ long. Leaves in the proximal half concentrate, then reduced and the most distal bracteiform; blades chartaceous in texture concolorous. Proximal leaves oblanceolate, oblong or elliptic, 4-7.5 × 1-2.5 cm, base attenuate, apex acute, one principal vein and 1-3 lateral veins, one medial and two lateral more or less basal, margins shortly denticulate, sparsely to densely pilose on both surfaces, covered by conical trichomes, with up to $800 \mu \mathrm{m}$ long; distal leaves reduced, elliptic or lanceolate, $1.5-3 \times 0.3-1.2 \mathrm{~cm}$, the most distal bractiform, elliptic-lanceolate or lanceolate, 0.5$1.5 \times 0.2-0.5 \mathrm{~cm}$. Heads disciform, solitary or up to 8 , peduncles $1.5-8 \mathrm{~cm}$ long, densely pilose; involucre 9-11 mm high, campanulate; phyllaries 3-4-seriate, gradate, margins pale, dorsally greenish and tips purplish when dry; outer phyllaries 4-6 × 0.7-0.9 mm, narrowly ellipticlanceolate or linear-lanceolate, acute, slightly pilose; middle and inner phyllaries 8-11 × 0.6-0.9 $\mathrm{mm}$, linear-elliptic, acute, dorsally scarcely pilose, margins deeply fimbriate at apex; receptacle subconvex, deeply alveolate. Ray flowers in 3-4-series, white-cream, then purplish in the end of the anthesis; corolla 7-8 $\mathrm{mm}$ long, tube 2-3 $\mathrm{mm}$, oblong, glabrous; style branches dorsally glabrous. Disc-flowers numerous, perfect, corolla 6-7 mm long, hairy with an ampliated throat, at apex 5-dentate, teeth $0.5-0.8 \mathrm{~mm}$; anthers $c$. $1.5 \mathrm{~mm}$ long, thecae auriculate at base, apical appendage oblong, subobtuse; style branches 1.5$2.0 \mathrm{~mm}$ long, dorsum with sweeping trichomes. Cypselae elliptic, 5-7 mm long, margins ribbed, narrowed at apex into a neck, neck 2-3 mm long; pappus c. $6.5-8 \mathrm{~mm}$ long, bristles unequal.

Etymology - The epithet Hieracifolia means "leaves of Hieracium L." genus of Compositae.

Material examined - BRAZIL. Rio Grande do Sul: "Bras. Province de Rio-Grande" 18161821, A. Saint-Hilaire 2650 (P02713426 photo!). Massambará, 7 Dec 2019, L.P. Deble \& B.P. Moreira 19005 (PACA!).

Discussion - The taxa Stenachaenium megapotamicum var. pedunculata and St. megapotamicum f. minor were proposed by Chodat (1902) and Chodat \& Hassler (1903). Later, Hassler (1919) evidenced both names as belonging to Podocoma Blanchetiana; however, St. megapotamicum $f$. minor was considered at variety level, under the name Podocoma Blanchetiana var. intermedia, due the general aspect of $P$. Blanchetiana and by its cypselae, with short neck, as found in $P$. hieracifolia. Sancho et al. (2010) treated Podocoma Blanchetiana var. intermedia as a synonym of $P$. hieracifolia, while the names proposed by Chodat were not mentioned. Later, St. megapotamicum var. pedunculata is recognized as valid by Chocarro \& De Egea (2018), and this variety and the f. minor are treated as valid for the Flora del Conosur (IBODA 2021). In the present study, all these names are recognized in the specific range of Podocoma hieraciifolia, one species that displays phenotypic variability in leaf shape and pilosity. I added two vouchers from Rio Grande do Sul because Sancho et al. (2010), in the last review of the genus not cited any material from this state.

\section{References}

Baker, J.G. 1882. Compositae. III. Asteroideae, Inuloideae. In: Martius, C.F.P. von \& Eichler, A.W. (Eds.) Flora Brasiliensis. Enumeratio plantarum in Brasilia, hactenus detectarum quas suis aliorumque botanicorum studiis descriptas et methodo naturali digestas partim icone illustratas, 6 (3). In Typographia Regia C. Wolf et fil. et in Offic. Lithograph. B. Keller. Monachii; apud Frid. Fleischer in comm., Lipsiae, pp. 1-134, tab. 1-44. https:// doi.org/10.5962/bhl.title.454

Bentham, G. \& J.D. Hooker. 1873. Genera plantarum ad exemplaria imprimis in herbariis Kewensibus servata definita, vol. 2(1). Lovell Reeve \& Co., Williams \& Norgate, Londini, 554 pp. https://doi.org/10.5962/bhl. title. 747

Cabrera, Á.L. 1932. Compuestas nuevas de la República Argentina. Notas Preliminares del Museo de La Plata 1 (21): 323-332.

Candolle, A.P. de. 1836. Prodromus Systematis Naturalis Regni Vegetabilis [. ..] Pars quinta: Systens Calycereas et Compositarum tribus priores. Sumptibus Sociorum Treuttel et Würtz, Parisiis, 706 pp. https://doi. org/10.5962/bhl.title. 286

Cassini, A.H.G. de. 1817. Aperçu des genres ou sous-genres nouveaux formés par M. Henri Cassini dans la famille des Synanthérées. Bulletin des Sciences, par la Société Philomatique: 137-140.

Cassini A.H.G. de. 1826. Podocome. In: Cuvier MF, ed. Dictionnaire des sciences naturelles dans lequel on traite méthodiquement des differents êtres de la nature, Vol. 42. Paris: Le Normant: 60-61.

Chocarro, M. del C.P. \& J. de Egea. 2018. Checklist of endemic vascular plants of Paraguay. Phytotaxa 384: 1-74. 
Deble, L.P., Updates in Podocoma.

Chodat. R. 1902. Plantae Hasslerianae soit Énumération des Plantes recoltees au Paraguay par le Dr. Emile Hasslerd d'Áarau (Suisse), de 1885 à 1895 et de 1898 à 1900 (à suivre). Bulletin de l'Herbier Boissier, ser. 2 , 2 (4): 382402.

Chodat. R. \& E. Hassler. 1903. Plantae Hasslerianae soit Énumération des Plantes recoltees au Paraguay par le Dr. Emile Hassler d'Áarau (Suisse), de 1885 à 1902 (à suite). Bulletin de l'Herbier Boissier, ser. 2, 3 (8): 701-732

Hassler, E. 1919. Ex herbario Hassleriano: Novitates paraguarienses. XXII. Repertorium Specierum Novarum Regni Vegetabilis. Centralblatt für Sammlung und Veroffentlichung von Einzeldiagnosen neuer Pflanzen 16: 25-29.

Hochreutiner, B.P.G. 1899. Compositae. In: Briquet J., Hochreutiner B.P.G, eds. VI Enumeration critique des plantes du Brésil meridional récoltées par E. M. Reineck et J. Czermak. Annuaire du Conservatoire \& du Jardin Botaniques de Genève 3: 147-175.
IBODA. 2021. Base de datos. Flora del Cono Sur. Instituto de Botánica Darwinion. Buenos Aires, Argentina. Available from: http:// www2.darwin.edu.ar/ (accessed 10 January 2021)

Malme, G.O. 1933. Compositae paranensis dusenianae. Kongl. Svenska Vetenskaps Academiens Handlingar. Stockholm 12: 1-122, pl. I-VI.

Nesom, G.L. 1994. Comments on Microgynella, Sommerfeltia, and Asteropsis (Asteraceae: Astereae). Phytologia 76: 101-105.

Nesom, G.L. \& D. Zanowiak. 1994. Taxonomic overview of Podocoma (Asteraceae, Astereae), with the incorporation of two species from Conyza. Phytologia 76: 106-114.

Sancho, G., N. Hind \& J.F. Prusky. 2010. Systematics of Podocoma (Asteraceae: Astereae): a generic reassessment. The Linnean Society of London, Botanical Journal of the Linnean Society 163: 486-513 